\title{
¿CUÁNTO IMPORTA EL ETIQUETADO ENERGÉTICO RESIDENCIAL EN LOS MERCADOS EMERGENTES?
Evaluación de las preferencias declaradas sobre sustentabilidad en el mercado inmobiliario de Santiago de Chile

\section{HOW MUCH DOES RESIDENTIAL ENERGY LABELLING MATTER IN EMERGING MARKETS? \\ Evaluation of the declared preferences on sustainability in the real estate market of Santiago de Chile}

\author{
ENCINAS, Felipe \\ Pontificia Universidad Católica de Chile \\ Facultad de Arquitectura, Diseño y Estudios Urbanos; Escuela de Arquitectura \\ Director de Investigación y Postgrado \\ El Comendador 1916, 7520245 Providencia, Santiago, Chile \\ Correo electrónico: felipe.encinas@uc.cl \\ Teléfono: +5622354 7721
}

\section{MARMOLEJO-DUARTE, Carlos}

Universidad Politécnica de Catalunya (UPC)

Departamento de Tecnología de la Arquitectura (TA), Centro de Política de Suelo y Valoraciones (CPSV)

Profesor Titular de Universidad

Av. Diagonal 649, 08028. Barcelona, España

Correo electrónico: carlos.marmolejo@upc.edu

Teléfono: +34 934054385

\section{AGUIRRE, Carlos}

Universidad de las Américas

Facultad de Arquitectura, Diseño y Construcción

Director Escuela de Construcción

Av. Manuel Montt 948, 7500972 Providencia, Santiago, Chile

Correo electrónico: caguirre@udla.cl

Teléfono: +56224275171

Palabras Clave: sustentabilidad; etiquetado energético; disposición a comprar; modelo Kano; Ingeniería Kansei

Key words: sustainability; energy labelling; willingness to buy; Kano model; Kansei Engineering

\section{Resumen}

El estudio de la relevancia de los atributos de eficiencia energética sobre las decisiones residenciales se ha realizado fundamentalmente desde las preferencias declaradas, puesto que la observación de éstos en transacciones reales es complicada. Dentro de estos estudios, los que abordan la temática desde una perspectiva integrada han cobrado particular relevancia, especialmente por el hecho de utilizar esquemas de certificación energética o ambiental como indicadores sintéticos de la globalidad de atributos. Ejemplo de esto son los etiquetados energéticos presentes en los Energy Performance Certificates (EPC) de la Unión Europea, introducidos con el fin de proveer transparencia energética a las transacciones de vivienda para 
fomentar decisiones de compra o arrendamiento mejor informadas. Efectivamente, los estudios aplicados a los EPC han demostrado que la eficiencia energética, medida mediante su certificación, puede cobrar relevancia por sobre otros atributos asociados a la calidad o al programa funcional de las viviendas. Sin embargo, esto no ha sido investigado en mercados emergentes -por ejemplo, en la región Latinoamericana- donde la aplicación de estos esquemas es reciente o han tenido una muy baja adopción por parte de los mercados inmobiliarios. Por esta razón, en esta comunicación se utiliza una metodología basada en la ingeniería emocional -específicamente a través de un modelo de Kano hibridado con Kansei Engineering- que busca establecer la percepción de los usuarios sobre los atributos de eficiencia energética, y en el fondo su utilidad percibida, en tres edificios residenciales de Santiago de Chile que poseen certificación energética. El objetivo de este artículo es identificar la relación que existe entre los atributos de eficiencia energética y la disposición a comprar, y la forma en cómo son percibidos por parte de una muestra de potenciales compradores de vivienda. La idea fundamental consiste en poder capturar, mediante técnicas cuantitativas, conceptos multidimensionales que derivan de la interacción de varios atributos inmobiliarios. A partir de una base de datos de 8.255 avisos de promoción inmobiliaria en prensa escrita entre 2012 y 2017 en Santiago de Chile, se establecieron 9 conceptos claves referidos a la eficiencia energética y sustentabilidad, más otros relativos al diseño y al concepto general de calidad, que se utilizan como argumento de venta. Estos son: [1] calidad; [2] buena inversión asociada a los atributos de sustentabilidad; [3] distinción con respecto a los demás; [4] buenos servicios comunes (ej. terraza, parrilla o piscina); [5] respeto por el medioambiente; [6] etiqueta de calificación energética como orientación para la decisión de compra; [7] ahorro energético; [8] seguridad; [9] ventanas de doble vidriado. De acuerdo con los resultados obtenidos, sólo los atributos de buena inversión, servicios comunes, ahorro energético, calidad, distinción y respeto por el medio ambiente aparecen significativamente correlacionados con la disposición a comprar, siendo los dos primeros particularmente relevantes (constituyendo lo que en el modelo de Kano se denominan "atributos de entusiasmo"). Sorprendentemente, la calificación energética incide negativamente en la disposición a comprar de los encuestados, lo que constituye uno de los hallazgos más relevantes. Esto no sólo contradice las conclusiones de la gran mayoría de los estudios aplicados en países desarrollados, sino que propone un gran desafío para la comunicación de una política pública definida como clave para la adopción masiva de la construcción sustentable en Chile. Los análisis multivariables aplicados sugieren que esta situación podría entenderse desde la percepción contrapuesta que existe entre los conceptos de confort térmico y de ahorro energético, asociado a la mala calidad térmica percibida de las envolventes en las viviendas. Empero, los atributos de sustentabilidad aparecen positivamente correlacionados con la disposición a comprar y, por ende, constituyen una clara señal para los desarrolladores puesto que son capaces de agregar valor a los productos inmobiliarios en tanto cuanto los hacen más atractivos.

\section{Abstract}

The relevance of residential energy efficiency attributes has mainly been studied from the perspective of stated preference methods, since it is difficult to analyse them in real transactions. The studies that have approached the subject from an integrated perspective have gained particular importance, especially when energy labelling schemes have been used as synthetic indicators for the totality of attributes. The European Energy Performance Certificates (EPC) of the Union constitute an example of this situation, since they were introduced to provide property transactions with energy transparency to encourage more informed buying or leasing

Citación: ENCINAS, F. et al. ¿Cuánto importa el etiquetado energético residencial en los mercados emergentes? Evaluación de las preferencias declaradas sobre sustentabilidad en el mercado inmobiliario de Santiago de Chile En: Libro de proceedings, CTV 2018. XII Congreso Internacional Ciudad y Territorio Virtual. "Ciudades y Territorios Inteligentes". UNCuyo, Mendoza, 5-7 septiembre 2018. Barcelona: CPSV, 2018, p. 285-302. 
decisions. Indeed, the studies applied to the EPC have shown that energy efficiency, as measured by its certification, can become relevant over other over traditional characteristics based on the quality of the finishings or the functional layout. However, this topic has not been investigated in emerging markets -e.g. the Latin American region- where the application of these schemes is recent or has had a very low adoption. This paper proposes a novel methodology based on a Kano model hybridized with Kansei Engineering to identify potential buyers' perception about energy efficiency attributes in three energy certified residential buildings in Santiago de Chile. The aim of this article is to identify the relationship between energy efficiency attributes and willingness to buy, and how they are perceived by a sample of buyers for new homes. The main idea is to capture -through quantitative techniquesmultidimensional concepts that derive from the interaction of many real estate attributes. Based on a database of 8,255 real estate advertisements between 2012 and 2017, 9 key concepts used as sales arguments- were established with respect to energy efficiency, sustainability, architectural design and construction quality. These are: [1] quality; [2] good investment related to sustainability; [3] distinction; [4] good services (e.g. terraces, barbeque area or pool); [5] environmentally friendly; [6] energy labelling as orientation for the purchasing decision; [7] energy saving; [8] security; [9] double glazed windows. According to the results, the attributes of "good investment related to sustainability", "good services", "energy savings", "quality", "distinction", and "environmentally friendly" appear significantly correlated with the willingness to buy. Additionally, the two first attributes are particularly relevant, which in the Kano model are called "attractive quality". Surprisingly, the energy labelling negatively affects the willingness to buy, which constitutes one of the most relevant findings of this research. Such result not only contradicts the conclusions of the vast majority of studies applied in the context of developed countries, but also implies a great challenge for a public policy that have been defined as key to encourage sustainable construction in Chile. The performed multivariate analyses suggest that this situation could be understood by the fact that users perceive the concept of thermal comfort as opposed to the energy savings, as consequence of the perceived poor thermal quality of housing envelopes. However, sustainability attributes are positive correlated to willingness to buy and, therefore, may be a clear signal for developers (since they are able to add value to real estate products).

\section{Introducción}

El estudio de la relevancia de los atributos de eficiencia energética sobre las decisiones residenciales se ha realizado fundamentalmente desde la perspectiva de las preferencias declaradas. Dichos estudios pueden clasificarse en dos grupos: 1) los que estudian de forma aislada algunos atributos arquitectónicos, es decir la sustitución puntual de dichos atributos en la actual vivienda y; 2) los que los estudian de forma integrada, es decir, como la adquisición o la compra, de viviendas ya edificadas y que generalmente utilizan esquemas existentes de certificación de la eficiencia energética/ambiental, como indicadores sintéticos de la globalidad de atributos arquitectónicos. Ejemplos del primer grupo son el trabajo de Poortinga, Steg, Vlek, \& Wiersma (2003) que han contrastado la importancia de emprender cambios en los sistemas de calefacción e iluminación frente a cambiar los hábitos de consumo energético. Dichos autores han encontrado que únicamente cuando la contribución marginal de los sistemas para

Citación: ENCINAS, F. et al. ¿Cuánto importa el etiquetado energético residencial en los mercados emergentes? Evaluación de las preferencias declaradas sobre sustentabilidad en el mercado inmobiliario de Santiago de Chile En: Libro de proceedings, CTV 2018. XII Congreso Internacional Ciudad y Territorio Virtual. "Ciudades y Territorios Inteligentes". UNCuyo, Mendoza, 5-7 septiembre 2018. Barcelona: CPSV, 2018, p. 285-302. 
con el consumo energético es evidente, entonces las personas los tienen en cuenta. Sadler (1998) encuentra que, si las estarían dispuestas a emprender reformas energéticas, sólo si la rentabilidad es alta, es decir, que puedan recuperar rápidamente el capital expenditure. El estudio de Banfi, Farsi, Filippini, \& Jakob (2008) ha demostrado que la disposición a pagar (DAP) por las mejoras energéticas depende de la percepción de mejora marginal, así las personas, por ejemplo, están más DAP por cambiar las ventanas que por aislar mejor las envolventes, entre otras cosas, porque entienden que podrían mejorar el confort acústico. Achtnicht \& Madlener (2014) encuentran que las reformas energéticas, únicamente pueden ocurrir, cuando es necesario sustituir los sistemas o emprender reformas funcionales. El segundo grupo de estudios es mucho más escaso, dentro del cual destacan dos trabajos. Heinzle, Boey Ying Yip, \& Low Yu Xing (2013) utilizan el análisis conjunto para determinar en qué medida los hogares en Singapur prefieren viviendas en función de su nivel de certificación según el esquema Green Mark. Sus resultados sugieren que otros atributos, tales como el precio, la superficie, la localización de la vivienda o la presencia de equipamientos son más importantes que la eficiencia ambiental de las viviendas globalmente mensurada. Marmolejo Duarte \& Bravi (2017) estudian con la misma metodología la relevancia relativa de la eficiencia energética en la elección de viviendas en alquiler y compra. Para ello, utilizan como indicador sintético tres diferentes niveles de eficiencia derivados de los Energy Performace Certificates (EPC). Sus resultados sugieren que, si las personas son informadas no sólo del ranking EPC, sino también de las repercusiones económicas y ambientales en unidades fácilmente comprensibles, entonces el atributo energético aparece como más relevante frente a otros relacionados con la calidad y el programa funcional de las viviendas.

\section{Regulaciones y certificación energética de viviendas en Chile}

El mercado de vivienda en Chile cuenta con una baja presencia del Estado - definido por López-Morales, Gasic Klett, \& Meza Corvalán (2012) como "urbanismo pro empresarial" - y citando a Savage, Warde, \& Ward (2003), "implica que no sólo que el Estado opera activamente en la producción de condiciones favorables para el mercado, sino que el mercado opera dentro del Estado con sus lógicas de competitividad y su dialéctica de volatilidad versus anclaje espacial del capital". Si bien la nueva Política Nacional Urbana (MINVU, 2014b) ha presentado elementos de gestión de las ciudades, ya en su diagnóstico se observa que el mercado inmobiliario está muy poco regulado, debido a que las externalidades propias de las nuevas urbanizaciones son entregadas a la compensación de los desarrolladores con muy pocas regulaciones. En esa lógica, la liberalización propia de los mercados chilenos, y en especial el de los mercados inmobiliarios, ha logrado que el estado haya sido reactivo, logrando establecer soluciones a los problemas ya existentes.

Pese a esto, Chile fue uno de los primeros países de la región en adoptar una regulación energética de carácter obligatorio para viviendas. En efecto, la denominada "reglamentación térmica", en el año 2000, estableció requerimientos de transmitancia térmica máxima admisible para techumbres, mientras que la actualización del año 2007 agregó exigencias de acondicionamiento térmico para techumbre, muros perimetrales y pisos ventilados (MINVU, 2016), en todo el territorio nacional. En efecto, de acuerdo al World Bank Working Paper No. 204, Chile - junto con México - parecían ser en ese momento los países más proactivos de Latinoamérica en relación a la eficiencia energética (Liu, Meyer, \& Hogan 2010). Sin embargo, de acuerdo a esta misma fuente, esta regulación puede ser considerada "relativamente permisiva en comparación con el estado del arte internacional". La OCDE también ha

Citación: ENCINAS, F. et al. ¿Cuánto importa el etiquetado energético residencial en los mercados emergentes? Evaluación de las preferencias declaradas sobre sustentabilidad en el mercado inmobiliario de Santiago de Chile En: Libro de proceedings, CTV 2018. XII Congreso Internacional Ciudad y Territorio Virtual. "Ciudades y Territorios Inteligentes". UNCuyo, Mendoza, 5-7 septiembre 2018. Barcelona: CPSV, 2018, p. 285-302. 
observado el asunto, recomendando encarecidamente que país pueda avanzar sobre mejores estándares de envolvente térmica, para "mejorar la calidad de la vivienda y proteger la salud pública" (Caldera Sánchez, 2012). En general, los expertos nacionales han valorado estos estándares como definición inicial, pero que al mismo tiempo están en deuda en términos de su aporte efectivo a la eficiencia energética de las viviendas (Bustamante, Cepeda, Martínez, \& Santa María, 2009; Bustamante, Rozas, Cepeda, Encinas, \& Martínez, 2009; Celis et al., 2012; Collados \& Armijo, 2008), además de no haber sido actualizada en 10 años. Estas debilidades de la regulación pueden ser entendidas por el hecho de estar definidas con el objetivo de "abordar solamente las condiciones mínimas para hogares promedio, o actuar como soluciones paliativas para edificios altamente inadecuados" (Bunster \& Noguchi, 2015). A raíz de esta situación, el Ministerio de Vivienda y Urbanismo impulsó una propuesta de actualización de la reglamentación térmica nacional en el año 2014, cuyos estándares representaban una considerable mejora en relación a la situación precedente, pasando - en el caso de Santiago de Chile - de un valor de $1.9 \mathrm{~W} / \mathrm{m}^{2} \mathrm{~K}$ a $0.6 \mathrm{~W} / \mathrm{m}^{2} \mathrm{~K}$ como transmitancia térmica máxima para muros (MINVU, 2014a). En términos constructivos, esto significaría pasar de un muro de albañilería de ladrillo sin aislación térmica a otro que debe incorporar al menos $50 \mathrm{~mm}$ (suponiendo poliestireno expandido de $20 \mathrm{~kg} / \mathrm{m}^{3}$ de densidad).

Al mismo tiempo, desde el año 2013 se ha implementado en Chile la "calificación energética", constituyéndose como un instrumento que evalúa la eficiencia energética de una vivienda, considerando su consumo de agua caliente sanitaria, iluminación y calefacción. Como resultado, se establece una etiqueta - que formalmente es muy similar al certificado energético aplicado por la Directiva Europea de Eficiencia Energética en las Edificaciones (Official Journal of the European Union, 2003) - y con un ranking en letras que va desde la "A" a la " $G$, ordenadas de más a menos eficiente y siendo la "E" la que representa el estándar constructivo contenido en la reglamentación térmica vigente. Para obtenerla, se estableció un mecanismo, que la mayoría de las veces hace uso de un procedimiento simplificado por medio de un cálculo en régimen estacionario. Si bien originalmente se contemplaba comenzar a implementar obligatoriamente el sistema de manera gradual a partir del 2016 (DITEC, 2015), esta fue postergada de manera indefinida, convirtiéndose en la práctica en voluntaria. Sin embargo, ésta ha presentado una adopción muy baja por parte del mercado. Por ejemplo, en términos de la calificación definitiva (vale decir, para viviendas con recepción municipal final obtenida), sólo un $5.4 \%$ de estas corresponden al mercado privado. Esto, en términos absolutos, se traduce en 594 viviendas en todo Chile desde el inicio del sistema en el 2013 hasta enero del 2017 (Ministerio de Vivienda y Urbanismo, 2017), lo cual es claramente marginal en comparación con el stock total del mercado inmobiliario, que a nivel nacional ha fluctuado entre las 67,781 y 101,398 unidades para el mismo periodo (CChC, 2017).

\section{Los modelos de Kano e Ingeniería Kansei en el estudio del rol que juegan los atributos inmobiliarios}

Según se ha dicho en la introducción la importancia relativa que los consumidores dan a los atributos de eficiencia energética es contingente a la percepción que tienen de estos y, en definitiva, a la utilidad percibida. Por tanto, en este artículo se acude al modelo de Kano, hibridado con el de Ingeniería Kansei, como vía para identificar: 1) la relación que existe entre los atributos de eficiencia energética y la disposición a comprar una vivienda, y 2) la forma en cómo son percibidos por parte de una muestra de potenciales compradores de vivienda.

Citación: ENCINAS, F. et al. ¿Cuánto importa el etiquetado energético residencial en los mercados emergentes? Evaluación de las preferencias declaradas sobre sustentabilidad en el mercado inmobiliario de Santiago de Chile En: Libro de proceedings, CTV 2018. XII Congreso Internacional Ciudad y Territorio Virtual. "Ciudades y Territorios Inteligentes". UNCuyo, Mendoza, 5-7 septiembre 2018. Barcelona: CPSV, 2018, p. 285-302. 
Por su parte la Ingeniería Kansei intenta aprender las percepciones, en su versión más emocional, que guían el comportamiento de los consumidores (Nagamachi, 1995). La idea fundamental es la de poder capturar, mediante técnicas cuantitativas, conceptos multidimensionales que derivan de la interacción de varios atributos. Por ejemplo, el concepto de "modernidad" de una vivienda, depende, por una parte, de los propios recursos cognitivos del consumidor, pero por otra, de la interacción de un conjunto de atributos individuales tales como el diseño, los materiales o los equipos con los que esté dotada. Conceptos como "sustentabilidad" o "respeto hacía el medio ambiente" relacionados con la eficiencia energética entran, como se ve, dentro de esta misma categoría de conceptos integrados por la interacción de varios atributos. Por su parte, el modelo de Kano intenta identificar el rol que juegan los atributos sobre la disposición a usar y en su caso, comprar o alquilar, un bien determinado (Kano, Seraku, Takahashi, \& Tsuji, 1984).

Dicho modelo supone que los atributos percibidos pueden adoptar tres roles fundamentales:

- Básicos: Son aquéllos cuya ausencia implica un rechazo en el uso/compra potencial del bien, si bien su presencia no incrementa el deseo de usarlo/comprarlo.

- Lineales: Son aquéllos cuya presencia guarda una correlación lineal con la disposición a usar/comprar le bien, cuánto mayor es la primera, mayor es la segunda.

- De entusiasmo: Son aquéllos cuya ausencia no incide sobre la disposición a usar/comprar el bien; si bien, su presencia desencadena un incremento significativo de dicha disposición.

Si bien la aplicación de las metodologías anteriores ha sido ampliamente utilizada en el ámbito de los bienes de consumo, también se han aplicación en la edificación y el urbanismo. Kinoshita, Cooper, Hoshino, \& Kamei (2004) utilizan Ingeniería Kansei para analizar el paisaje cromático de los entornos edificados. Mientras que Jennings \& Cain (2013) en el diseño de paisajes sonoros y Lee, Jang, Wang, \& Namgung (2009) en el diseño de calles. Llinares \& Page $(2007,2011)$ dan un paso adelante para hibridar, desde la perspectiva de la aplicación práctica, ambas técnicas, y aplicarlas al estudio de las percepciones que, sobre los posibles compradores, generan los nuevos desarrollos residenciales. Así, encuentran que conceptos como la durabilidad, el diseño/elegancia, o la calidad son determinantes de la disposición a comprar; siendo que algunos son de tipo básico (ej. privacidad), otros lineales (ej. durabilidad) y otros de entusiasmo (ej. elegancia). Esta misma hibridación es usada por Marmolejo-Duarte \& Villar Llull (2015) en el estudio de las transformaciones urbanas. Como se ve, ninguno de los trabajos ha abordado aún las dimensiones de la eficiencia energética y la sustentabilidad, como se hace en el presente según se explica a continuación.

\section{Metodología}

En este trabajo se utiliza la aproximación hibrida del modelo de Kano y de Ingeniería Kansei establecido en el estudio ya mencionado de Llinares \& Page $(2007,2011)$ y en el de Tama, Azlia, \& Hardiningtyas (2015) para otro tipo de aplicaciones. Dicha variante requiere la aplicación de los siguientes pasos:

\subsection{Identificación de las Kansei Words y construcción de la encuesta}

Se trata de identificar las palabras que mejor describen los conceptos cuya percepción será evaluada, las cuales toman la forma de adjetivos, sustantivos, verbos y en ciertos casos sentencias (Nagamachi, 2011). Estas, por lo general, son recolectadas desde revistas de

Citación: ENCINAS, F. et al. ¿Cuánto importa el etiquetado energético residencial en los mercados emergentes? Evaluación de las preferencias declaradas sobre sustentabilidad en el mercado inmobiliario de Santiago de Chile En: Libro de proceedings, CTV 2018. XII Congreso Internacional Ciudad y Territorio Virtual. "Ciudades y Territorios Inteligentes". UNCuyo, Mendoza, 5-7 septiembre 2018. Barcelona: CPSV, 2018, p. 285-302. 
amplia difusión, periódicos de negocios o de información de agentes de ventas con respecto a la percepción de los consumidores y requieren un proceso de síntesis para seleccionar finalmente las Kansei Words más relevantes. Para este trabajo se han estudiado la totalidad de los anuncios publicitarios de promoción inmobiliaria en Santiago de Chile aparecidos en la revista "Vivienda Decoración" de El Mercurio y el suplemento "Casas" de Publimetro, entre enero de 2012 y julio de 2017. El primero corresponde a un periódico de circulación nacional, mientras que el segundo es de entrega gratuita y está restringido a la Región Metropolitana de Santiago.

En total, se catastraron 8255 avisos publicitarios, construyendo una bases de datos, que en su análisis descriptivo y longitudinal fue publicada en Encinas \& Aguirre (2017). A partir de ésta, se identificaron todos los atributos inmobiliarios contenidos en la publicidad, tanto los declarados explícitamente (por medio del texto), como los sugeridos mediante las imágenes.

En base a esto anterior, se identificaron 9 conceptos claves relativos a la sustentabilidad, y más particularmente los relacionados con la eficiencia energética, que suelen utilizarse como argumento de venta en el ámbito de estudio, que aparecían con mayor frecuencia en desarrollos de edificios en altura y en las comunas con mayor presencia de oferta inmobiliaria dentro de la ciudad de Santiago. En este contexto, las 9 Kansei Words seleccionadas para caracterizar la oferta desde esta perspectiva - más otros relativos al diseño y el concepto general de calidad - fueron las siguientes: (1) calidad; (2) buena inversión asociada a los atributos de sustentabilidad; (3) distinción con respecto a los demás; (4) buenos servicios comunes (ej. quincho, terraza o piscina); (5) respeto por el medioambiente; (6) etiqueta de calificación energética como orientación para la decisión de compra; (7) ahorro energético; (8) seguridad; (9) ventanas de doble vidriado.

Debido a que estas 9 Kansei Words eran mejor expresadas en términos de sentencias por sobre pares de adjetivos opuestos, se optó por construir la encuesta en base a éstas. Si bien la aproximación habitualmente utilizada en Ingeniería Kansei es la del diferencial semántico (Nagamachi, 2011), la alternativa propuesta resulta consistente con la idea de que cada par de palabras constituyen un "continuum" (Ishihara, 2011), el cual en este caso se expresa en una sentencia. Esta también resulta apropiada en la hibridación con el modelo de Kano donde la adecuada categorización de los atributos debería estar basada en la provisión (o no provisión) de éste, por sobre criterios de desempeño (Mikulić \& Prebežac, 2011).

Con los atributos antes identificados, se ha construido una encuesta, en dónde las personas respondientes tenían que decir, en una escala tipo Likert, cuan de acuerdo estaban con la presencia de dichos atributos en los estímulos presentados, tal como se presenta en la Tabla 1. Se ha eliminado deliberadamente la respuesta neutral dentro la escala, con la intención de obligar a tomar una posición por parte de los encuestados. En dicha encuesta también se han incluido características de índole sociodemográfica (edad y nivel educacional), así como la autopercepción de conocimiento acerca de la certificación energética de viviendas chilena. Finalmente, de manera similar al estudio de Llinares \& Page (2011), se ha agregó al cuestionario una variable de evaluación global acerca de la disposición a comprar, por medio de la siguiente expresión: "Suponiendo que su presupuesto se lo permite y que encuentra el departamento que busca. En una escala de 1 a 7 (donde 1 es menos y 7 es más), ¿Cuán dispuesto estaría a comprar un departamento en este edificio?"

Citación: ENCINAS, F. et al. ¿Cuánto importa el etiquetado energético residencial en los mercados emergentes? Evaluación de las preferencias declaradas sobre sustentabilidad en el mercado inmobiliario de Santiago de Chile En: Libro de proceedings, CTV 2018. XII Congreso Internacional Ciudad y Territorio Virtual. "Ciudades y Territorios Inteligentes". UNCuyo, Mendoza, 5-7 septiembre 2018. Barcelona: CPSV, 2018, p. 285-302. 
Tabla 1. Configuración de las preguntas sobre percepción de atributos inmobiliarios a partir de metodología Ingeniería Kansei y modelo de Kano

\begin{tabular}{|c|c|c|c|c|c|}
\hline & & $\begin{array}{l}\text { Muy de } \\
\text { acuerdo }\end{array}$ & $\begin{array}{l}\text { Algo de } \\
\text { acuerdo }\end{array}$ & $\begin{array}{c}\text { Algo en } \\
\text { desacuerdo }\end{array}$ & $\begin{array}{c}\text { Muy en } \\
\text { desacuerdo }\end{array}$ \\
\hline 1 & "Este edificio es de buena calidad" & & & & \\
\hline 2 & "Este edificio parece térmicamente confortable " & & & & \\
\hline 3 & $\begin{array}{l}\text { "Este edificio es una buena inversión debido a sus } \\
\text { características de sustentabilidad" }\end{array}$ & & & & \\
\hline 4 & "Este edificio se distingue del resto" & & & & \\
\hline 5 & $\begin{array}{l}\text { "Este es un buen edificio en términos de sus servicios } \\
\text { comunes" }\end{array}$ & & & & \\
\hline 6 & $\begin{array}{l}\text { "En este edificio viviría una persona respetuosa con el medio } \\
\text { ambiente" }\end{array}$ & & & & \\
\hline 7 & $\begin{array}{l}\text { "La etiqueta energética es una buena ayuda para la decisión } \\
\text { de compra" }\end{array}$ & & & & \\
\hline 8 & "Este edificio permite ahorrar en la cuenta de luz y gas" & & & & \\
\hline 9 & $\begin{array}{l}\text { "Este edificio tiene ventanas que reducen el consumo de } \\
\text { energía" }\end{array}$ & & & & \\
\hline
\end{tabular}

Fuente: Elaboración propia

\subsection{Aplicación de la encuesta de percepción de atributos inmobiliarios de sustentabilidad}

A diferencia de lo que es común en la aplicación de estas técnicas, los estímulos en este caso no son teóricos ni reproducidos, sino que derivan de promociones inmobiliarias reales del mercado residencial de Santiago de Chile. En coherencia con esta situación y dado que el objetivo de esta exploración es indagar en la percepción emocional de atributos inmobiliarios de sustentabilidad, se escogieron tres desarrollos inmobiliarios que fueran representativos del mercado residencial privado en Santiago en cuanto a su aproximación a estos atributos. Como ya se mencionó, el mercado de vivienda plurifamiliar fue el escogido para el análisis, dado su masividad e impacto en la construcción de la ciudad. Al mismo tiempo, se seleccionaron proyectos asociados a segmentos de usuarios de renta media, donde los argumentos de reducción en el consumo (gasto) en calefacción, electricidad o agua caliente sanitaria, asociados a varios de los atributos inmobiliarios de eficiencia energética, resultan particularmente relevantes, tal como está descrito en Encinas, Marmolejo, \& Aguirre (2016). En el contexto nacional, estos segmentos pueden asociarse a rangos de precios de oferta entre 1000 y 4000 UF $^{1}$, que corresponden a valores entre US $\$ 41,000$ y US $\$ 161,000$. Por sobre este último valor, se ha observado que varios de estos atributos comienzan a formar parte del estándar constructivo esperable (por ejemplo, el doble vidriado) por lo que su capacidad diferenciadora disminuye, apareciendo otros que apelan a una dimensión más valórica de la sustentabilidad, implicando recompensas de tipo psicosocial (DEFRA, 2007; Lippincott Mercer, 2006).

En consecuencia, la encuesta fue aplicada a los posibles compradores de 3 proyectos inmobiliarios (edificios de departamentos), ubicados en Santiago (Figura 1), y que habían acudido por muto propio a las salas de venta. Estos 3 proyectos han sido calificados

1 La Unidad de Fomento (UF) es una unidad de cuenta utilizada en Chile, la cual se ajusta de acuerdo con la inflación y que es utilizada ampliamente para determinar los costos de construcción, valores de las viviendas y créditos hipotecarios. De acuerdo a Central Bank of Chile (2018), 1 UF $=$ US $\$ 40.7$ 
energéticamente y representan nichos de mercado similares, de acuerdo a las características presentadas en la Tabla 2. Luego, una vez que las personas participantes habían sido informadas sobre las promociones, y habían visitado el "departamento piloto", entonces se les pedía, con la intervención de un encuestador presencial, que respondiesen la encuesta. Al finalizar la encuesta los respondientes tenían que declarar la intensidad con la que estarían dispuestos a comprar la vivienda en el caso de que su precio les resultara asequible.

Figura 1. Ubicación de los 3 proyectos estudiados en el área metropolitana de Santiago

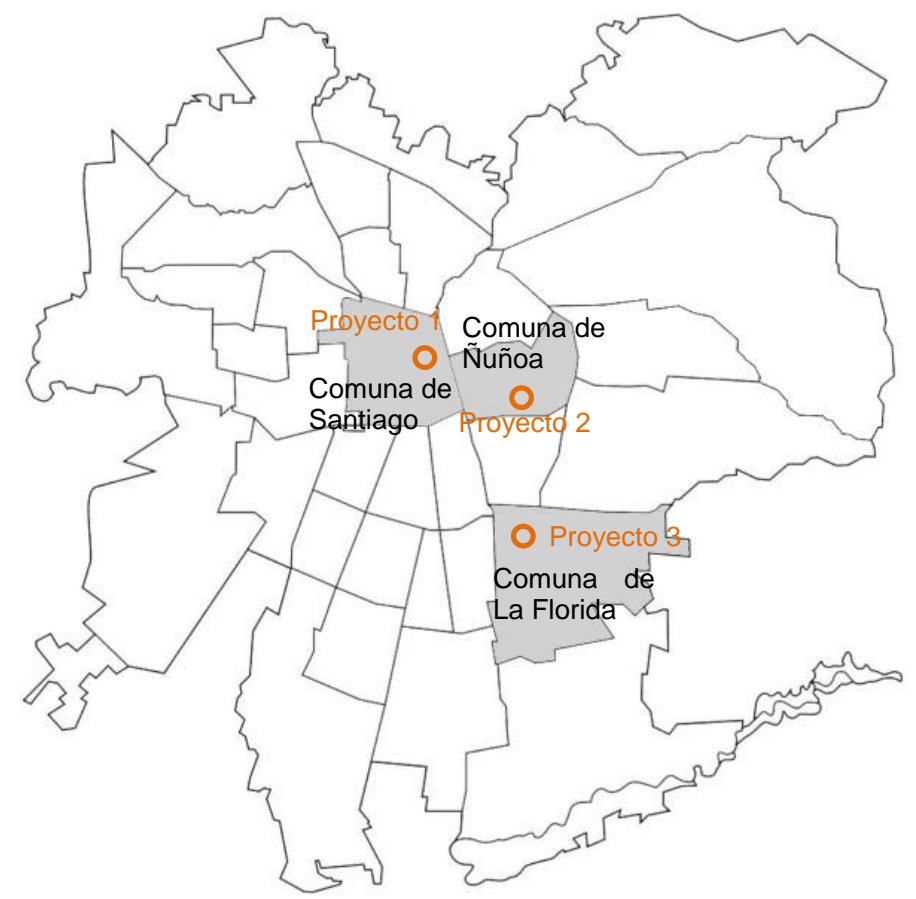

Fuente: Elaboración propia

Tabla 2: Descripción de los 3 proyectos inmobiliarios en los cuales se aplicó la encuesta

\begin{tabular}{|c|c|c|c|}
\hline & Proyecto 1 & Proyecto 2 & Proyecto 3 \\
\hline Comuna & Santiago & Ñuñoa & La Florida \\
\hline Número de departamentos & 250 & 20 & 144 \\
\hline Número de plantas & 16 & 5 & 18 \\
\hline Precio menor (desde) & US $\$ 90,000$ & US $\$ 200,000$ & US $\$ 150,000$ \\
\hline Precio mayor (hasta) & - & US $\$ 350,000$ & - \\
\hline Superficie útil menor (desde) & $37 \mathrm{~m}^{2}$ & $62 \mathrm{~m}^{2}$ & $60 \mathrm{~m}^{2}$ \\
\hline Superficie útil mayor (hasta) & $59 \mathrm{~m}^{2}$ & $120 \mathrm{~m}^{2}$ & $83 \mathrm{~m}^{2}$ \\
\hline Número máximo de dormitorios & 2 & 3 & 3 \\
\hline Número máximo de baños & 2 & 2 & 2 \\
\hline Tipos de departamentos & Normal, dúplex, triplex & Normal, dúplex, triplex & Normal \\
\hline Calificación energética obtenida & $D$ & Entre letras $A$ y $G$ & $\begin{array}{l}\text { Calificada, pero no } \\
\text { declarada }\end{array}$ \\
\hline Atributos de sustentabilidad & $\begin{array}{l}\text { Colectores solares } \\
\text { térmicos, paneles } \\
\text { fotovoltaicos, aislación } \\
\text { térmica, artefactos } \\
\text { eficientes para el } \\
\text { ahorro de agua }\end{array}$ & $\begin{array}{l}\text { Aislación térmica, } \\
\text { equipamiento "full } \\
\text { electric", doble } \\
\text { vidriado en ventanas, } \\
\text { aperturas según } \\
\text { orientación }\end{array}$ & $\begin{array}{l}\text { Colectores solares } \\
\text { térmicos, aislación térmica } \\
\text { por el exterior (EIFS), } \\
\text { doble vidriado en ventanas, } \\
\text { artefactos eficientes para el } \\
\text { ahorro de agua }\end{array}$ \\
\hline
\end{tabular}

Fuente: Elaboración propia

Citación: ENCINAS, F. et al. ¿Cuánto importa el etiquetado energético residencial en los mercados emergentes? Evaluación de las preferencias declaradas sobre sustentabilidad en el mercado inmobiliario de Santiago de Chile En: Libro de proceedings, CTV 2018. XII Congreso Internacional Ciudad y Territorio Virtual. "Ciudades y Territorios Inteligentes". UNCuyo, Mendoza, 5-7 septiembre 2018. Barcelona: CPSV, 2018, p. 285-302. 


\subsection{Aplicación de modelo de Kano y de Ingeniería Kansei con los resultados de la encuesta}

Para cada uno de los atributos (Kansei Words) evaluados se ha realizado una recodificación. De forma que cuando el respondiente percibía que un atributo estaba perfectamente ausente en la promoción inmobiliaria utilizada como estímulo, entonces se adopta un valor de -2; por el contrario, si el respondiente percibía que el atributo estaba perfectamente presente en dicha promoción, entonces se adopta un valor de 2 . Siendo que las situaciones intermedias adoptan el valor de $-1 \mathrm{y}+1$. En definitiva, esta recodificación permite ordenar las percepciones de ausencia/presencia de cada atributo. A partir de esta información se ha construido el Modelo de Kano y su espacio derivado. Para ello se ha calculado la correlación de Spearman entre la percepción de presencia/ausencia de cada atributo y la disposición general a comprar la vivienda. De forma que los atributos cuyas correlaciones tienen el signo esperado:

a. y son significativas cuando el atributo está ausente, pero no cuando está presente, son de tipo "básico"

b. y son significativas tanto cuando el atributo está ausente como presente, son de tipo "lineal"

c. y son significativas cuando el atributo está presente, pero no cuando está ausente, son de tipo "de entusiasmo"

En virtud de que es posible que atributos como "ahorro energético" o "respeto al medio ambiente" estén correlacionados se ha realizado un análisis de componentes principales. Que, además de eliminar la redundancia de información, ha permitido encontrar las dimensiones latentes en la percepción de los encuestados. La representación del espacio factorial resultante, en relación a la disposición de compra de la vivienda en cuestión, permite establecer asociaciones que permitan interpretar los resultados.

\section{Resultados y discusión}

En el análisis del espacio de Kano es necesario definir los lugares geométricos, sobre los cuales se pueden establecer las relaciones entre los atributos y su impacto en la decisión de compra del producto inmobiliario.

En esa lógica, se presenta la figura ํo2, modificada de Kano et al. (1984); Llinares \& Page (2011) y Violante \& Vezzetti (2017). En ella se observan, cinco espacios geométricos donde se ubicarían los distintos atributos, siendo categorizados como básicos, lineales, de entusiasmo y revertidos. En la figura $\mathrm{N}^{\circ} 3$ se establecen los resultados del análisis. Los atributos básicos identificados, aunque no son significativos al $90 \%$, fueron los atributos asociadas a la eficiencia de la vivienda, además de la seguridad, entendida como la seguridad ante actos delictivos del departamento. En sí este punto, no es sorprendente ya que son elementos que normalmente son evidenciados por la publicidad (Encinas, Aguirre, \& Marmolejo-Duarte, 2018). Por otro lado, los atributos lineales, o sea los que aumenta el atractivo en la medida que aumentan su presencia están asociados a la percepción de calidad del producto, el respeto al medio ambiente como valor, el ahorro energético y la distinción como atributo arquitectónico. Esto es, sin lugar a dudas, pertinente para entender cuál es el imaginario del comprador, en ambos mercados identificando la vivienda como logro, y como la compra de este bien permite la manifestación física del logro.

Citación: ENCINAS, F. et al. ¿Cuánto importa el etiquetado energético residencial en los mercados emergentes? Evaluación de las preferencias declaradas sobre sustentabilidad en el mercado inmobiliario de Santiago de Chile En: Libro de proceedings, CTV 2018. XII Congreso Internacional Ciudad y Territorio Virtual. "Ciudades y Territorios Inteligentes". UNCuyo, Mendoza, 5-7 septiembre 2018. Barcelona: CPSV, 2018, p. 285-302. 
Figura 2. Modificación propuesta del modelo de Kano original a partir de la información recogida por la encuesta

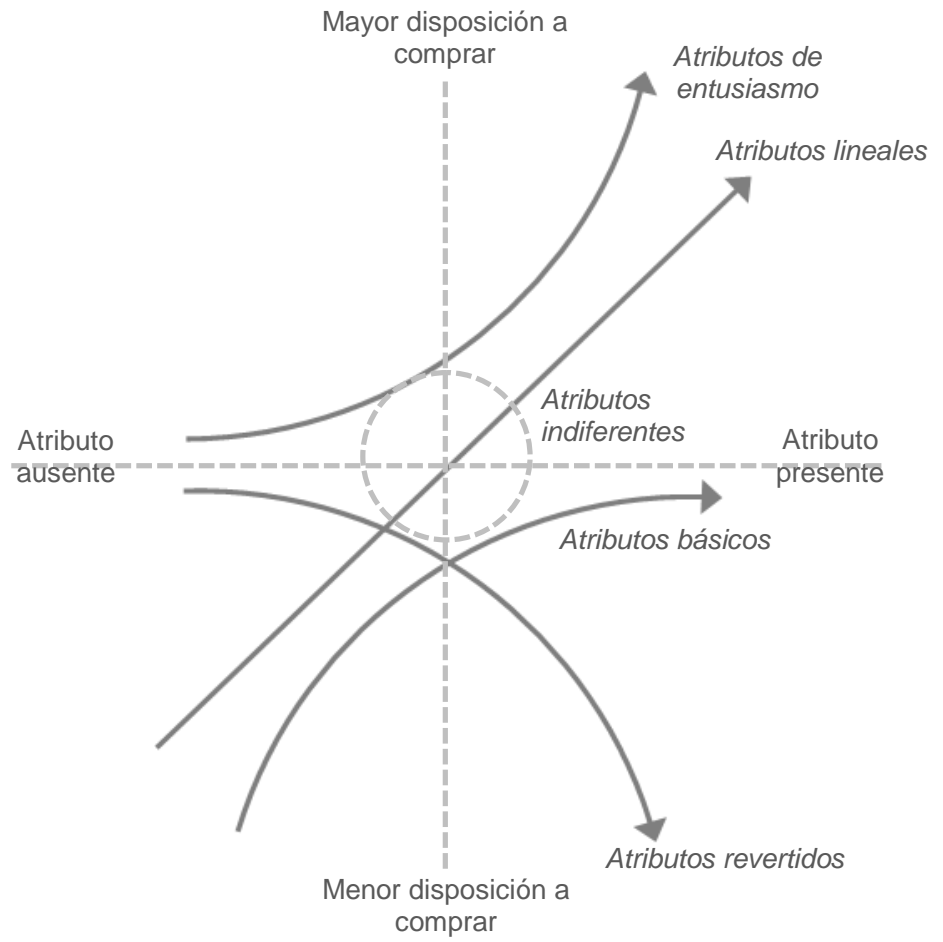

Fuente: Elaboración propia

Figura 3. Correlación de Spearman (rho) entre atributos presentes y ausentes contenidos en la encuesta con respecto a la disposición a comprar, para la definición del Espacio de Kano

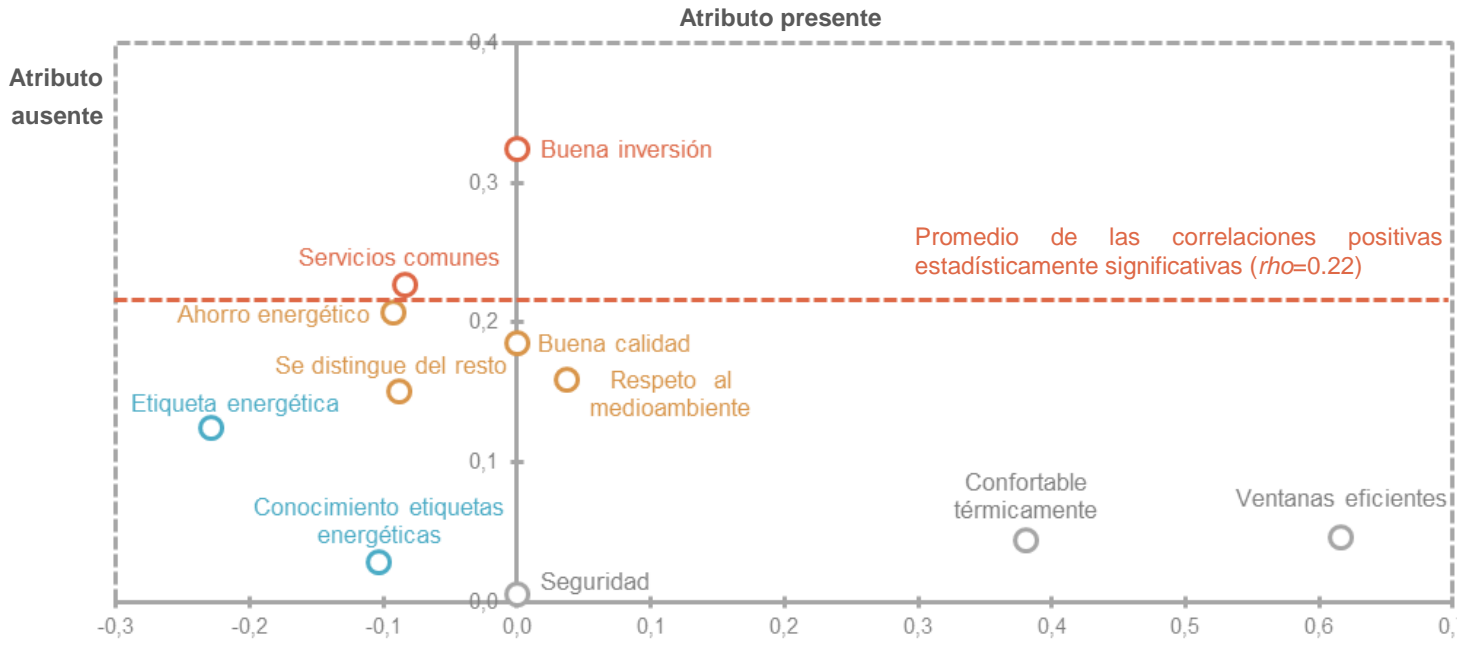

Atributos contenidos en la encuesta:

Sólo presente (sobre el promedio de las correlaciones positivas)

Sólo presente (bajo el promedio de las correlaciones positivas)

Sólo ausente

No estadísticamente significativo para $p<0.09$

Fuente: Elaboración propia

Citación: ENCINAS, F. et al. ¿Cuánto importa el etiquetado energético residencial en los mercados emergentes? Evaluación de las preferencias declaradas sobre sustentabilidad en el mercado inmobiliario de Santiago de Chile En: Libro de proceedings, CTV 2018. XII Congreso Internacional Ciudad y Territorio Virtual. "Ciudades y Territorios Inteligentes". UNCuyo, Mendoza, 5-7 septiembre 2018. Barcelona: CPSV, 2018, p. 285-302. 
Por otro lado, y siguiendo en esta línea, los atributos de entusiasmo corresponden a la percepción de una buena inversión (o sea un bien rentable de largo plazo, con flexibilidad asociada a su explotación), por un lado y el estándar de los servicios comunes (piscinas, salones comunes, quinchos y terrazas), por el otro. Estos elementos muestran una importante influencia en la decisión de compra, por sobre los otros atributos.

Los atributos asociados a la calificación energética, asociada a las letras obtenidas, y el conocimiento de este proceso, resultan atributos revertidos, o sea que entre más presencia tienen, disminuyen la atracción del producto. En este punto es necesario detenerse, y establecer dos posibles explicaciones preliminares; la primera guarda relación con el conocimiento técnico de lo que significa la calificación, y por ende, no poder incorporar a la decisión de compra los elementos de juicio adecuados. En ese sentido, asumiendo que hoy en Chile, la principal calificación de eficiencia energética corresponde a la calificación de electrodomésticos, donde mayor letra es también mayor percepción de precio, se puede transmitir a la vivienda ante un sistemático desconocimiento de la verdadera dimensión de la calificación de vivienda. Es por eso, que la calificación de vivienda percibida como variable correlacionada con el precio de la vivienda, hace que tenga una percepción de menor atracción de compra. En palabras simples, "como sé que es mejor, debe ser más cara". Una segunda explicación preliminar, guarda relación con la escasa percepción de la certificación como medida de mejora térmica de la vivienda.

Para buscar explicaciones más certeras sobre estos atributos revertidos, se procedió a realizar un análisis de componentes principales sobre las 11 variables que representaban los diversos atributos. En ese sentido, la Tabla 3 y la Figura 4 presentan los resultados de este análisis. Las variables originales se sedimentan en 4 componentes independientes, percepción emocional [C1], percepción del confort y características constructivas [C2], etiquetado energético y nivel de estudios [C3], y la percepción racional [C4]. En una primera instancia, la existencia de una variable donde sedimente el nivel de conocimiento sobre el etiquetado energético, el nivel educacional, y la valoración de las etiquetas, es en sí un hallazgo importante. En ese sentido y por sí mismo, esto representa que existe una relación directamente proporcional entre estos elementos, a tal nivel que se encuentran auto correlacionados. En sí, esta percepción resulta independiente de las otras.

En el caso de los dos primeros componentes [C1] y [C2], la percepción emocional y el confort y características constructivas, los atributos de etiqueta energética y nivel de estudios se encuentran de forma positiva en la apreciación de la dimensión de confort, sin embargo, el etiquetado, se presenta en el cuadrante negativo. Esto potenciaría la explicación anterior, donde el etiquetado, se presentan como variable relacionada con la percepción de mayor precio. Al cruzar los componentes [C1] y [C3], la percepción emocional y el conocimiento del etiquetado y nivel de estudios, se observa que las variables de confort y calidad de la construcción, resultan poco relacionadas con el ahorro energético y con una percepción emocional distinta a la etiqueta como elemento de decisión de compra de vivienda. Este punto sigue aportando una explicación razonable para la caracterización de los atributos revertidos para las etiquetas energéticas.

Por último, el análisis de los componentes [C1] de percepción emocional y [C4] de percepción racional, (o valoración de los ahorros y la eficiencia), presentan sentidos distintos en las variables que estamos investigando, por un lado, el conocimiento declarado de las etiquetas, junto con el nivel de estudios, y en sentido inverso la etiqueta energética como ayuda a la decisión de compra. Esto nuevamente valida la explicación preliminar de que, a menor 
conocimiento de la etiqueta energética y menor nivel de estudios, existe una decisión más emocional y negativa hacia la etiqueta como mejora en las condiciones de la vivienda a comprar. De la misma forma, el etiquetado, presenta un componente más racional, pero en la decisión de compra, el componente emocional no lo logra posicionar en el mismo cuadrante de los atributos de confort, ahorro, calidad, etc.

\section{Tabla 3. Cargas factoriales en matriz rotada con resultados de Análisis de Componentes Principales* para 11 variables}

\begin{tabular}{|c|c|c|c|c|}
\hline & $\begin{array}{l}\text { [C1] } \\
\text { Percepción } \\
\text { emocional }\end{array}$ & $\begin{array}{c}\text { [C2] } \\
\text { Confort y } \\
\text { características } \\
\text { constructivas }\end{array}$ & $\begin{array}{l}\text { [C3] Etiquetas } \\
\text { energéticas y } \\
\text { educación }\end{array}$ & $\begin{array}{l}\text { [C4] } \\
\text { Percepción } \\
\text { racional }\end{array}$ \\
\hline "Este edificio es de buena calidad" & 0.56 & & & \\
\hline "Este edificio parece térmicamente confortable " & & 0.72 & & \\
\hline $\begin{array}{l}\text { "Este edificio es una buena inversión debido a sus } \\
\text { características de sustentabilidad" }\end{array}$ & 0.59 & & & \\
\hline "Este edificio se distingue del resto" & 0.60 & & & \\
\hline $\begin{array}{l}\text { "Este es un buen edificio en términos de sus servicios } \\
\text { comunes" }\end{array}$ & & & & 0.72 \\
\hline $\begin{array}{l}\text { "En este edificio viviría una persona respetuosa con } \\
\text { el medio ambiente" }\end{array}$ & 0.72 & & & \\
\hline $\begin{array}{l}\text { "La etiqueta energética es una buena ayuda para la } \\
\text { decisión de compra" }\end{array}$ & & & 0.72 & \\
\hline $\begin{array}{l}\text { "Este edificio permite ahorrar en la cuenta de luz y } \\
\text { gas" }\end{array}$ & & & & 0.69 \\
\hline $\begin{array}{l}\text { "Este edificio tiene ventanas que reducen el consumo } \\
\text { de energía" }\end{array}$ & & 0.85 & & \\
\hline Nivel de conocimiento sobre etiquetas energéticas & & & 0.52 & \\
\hline Nivel de estudios & & & 0.53 & \\
\hline Autovalores & 2.35 & 1.45 & 1.10 & 1.07 \\
\hline Porcentaje de la varianza & $21.3 \%$ & $13.2 \%$ & $10.0 \%$ & $9.8 \%$ \\
\hline Varianza acumulada & $21.3 \%$ & $34.5 \%$ & $44.5 \%$ & $54.3 \%$ \\
\hline
\end{tabular}

Method of rotation: Varimax with Kaiser normalisation.

Nota $\left({ }^{*}\right)$ : Componentes con autovalores $<1.0$ fueron suprimidos de la solución final de acuerdo al criterio de Kaiser. Cargas factoriales $>|0.5|$ fueron consideradas como significativas de acuerdo al criterio de muestreo establecido por Hair, Anderson, Tatham, \& Black (2005, p. 100). Los valores en la diagonal de la matriz de correlación anti-imagen y las comunalidades están todas sobre 0.5 , lo que confirma que cada variable comparte al menos la mitad de la varianza con otras variables.

Fuente: Elaboración propia

\section{Conclusiones}

Esta investigación busca establecer cuál sería el aporte a la decisión de compra o atracción de un etiquetado energético, para un producto inmobiliario. Mediante la metodología de Ingeniería Kansei, aplicada a 3 edificios en la ciudad de Santiago de Chile, se estableció, que primero los atributos de confort térmico y etiquetado energético están en diferentes posiciones del Espacio de Kano desarrollado. Mientras los primeros son básicos (y estadísticamente no significativos), los atributos de etiquetado son revertidos, o sea se presentan como negativos ante la decisión de compra. Además, el análisis de componentes principales, explica aún más esta situación que puede ser resumida como un desacople en la percepción de lo que efectivamente se está midiendo en el etiquetado, como también, en el aporte de estos a la elección.

Citación: ENCINAS, F. et al. ¿Cuánto importa el etiquetado energético residencial en los mercados emergentes? Evaluación de las preferencias declaradas sobre sustentabilidad en el mercado inmobiliario de Santiago de Chile En: Libro de proceedings, CTV 2018. XII Congreso Internacional Ciudad y Territorio Virtual. "Ciudades y Territorios Inteligentes". UNCuyo, Mendoza, 5-7 septiembre 2018. Barcelona: CPSV, 2018, p. 285-302. 
Figura 4. Mapas de variables según cruces de componentes para [C1], [C2], [C3] y [C4]
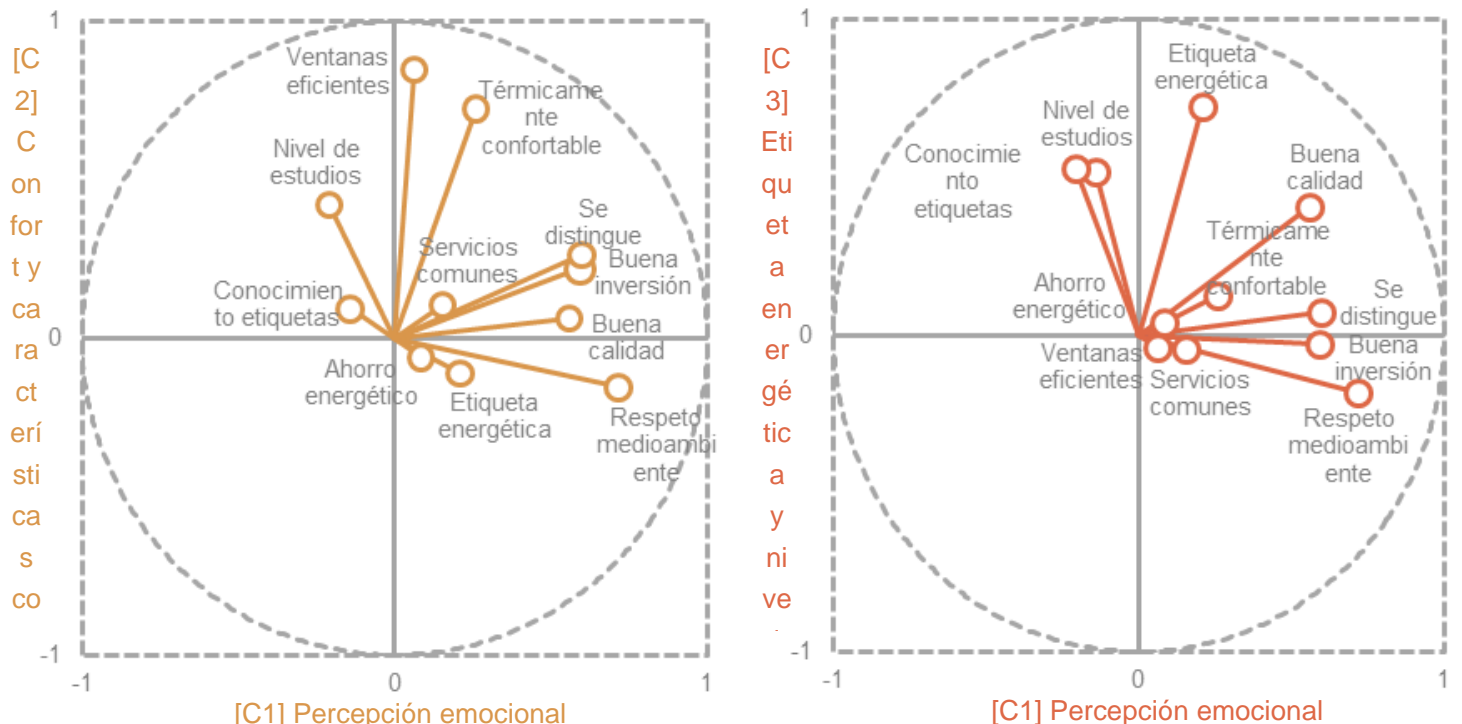

[C1] Percepción emociona

[C1] Percepción emocional

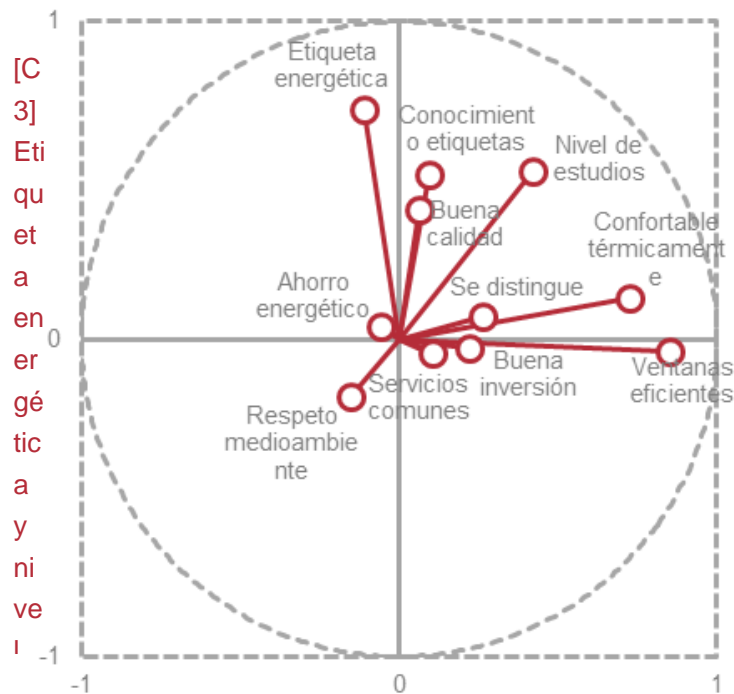

[C2] Confort y características constructivas

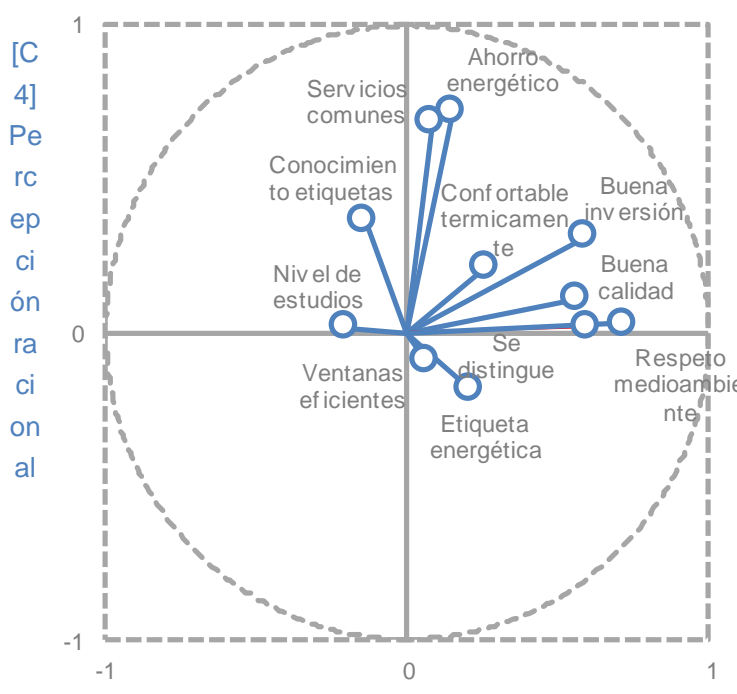

[C1] Percepción emocional

Fuente: Elaboración propia

\section{Bibliografía}

ACHTNICHT, M. \& MADLENER, R. Factors influencing German house owners' preferences on energy retrofits. En: Energy Policy, Mayo 2014, 68: 254-263. [En línea] Disponible en: $\quad$ https://www.sciencedirect.com/science/article/pii/S0301421514000111 DOI: http://doi.org/10.1016/j.enpol.2014.01.006

BANFI, S.; FARSI, M.; FILIPPINI, M. \& JAKOB, M. Willingness to pay for energy-saving measures in residential buildings. En: Energy Economics, Marzo 2008, 30 (2): 503-516. [En línea] Disponible en: https://www.sciencedirect.com/science/article/pii/S0140988306000764 DOI: http://doi.org/10.1016/j.eneco.2006.06.001

Citación: ENCINAS, F. et al. ¿Cuánto importa el etiquetado energético residencial en los mercados emergentes? Evaluación de las preferencias declaradas sobre sustentabilidad en el mercado inmobiliario de Santiago de Chile En: Libro de proceedings, CTV 2018. XII Congreso Internacional Ciudad y Territorio Virtual. "Ciudades y Territorios Inteligentes". UNCuyo, Mendoza, 5-7 septiembre 2018. Barcelona: CPSV, 2018, p. 285-302. 
BUNSTER, V. \& NOGUCHI, M. Profiling Space Heating Behavior in Chilean Social Housing: Towards Personalization of Energy Efficiency Measures. En: Sustainability, 2015, 7 (6): 79737996. [En línea] Disponible en: https://www.mdpi.com/2071-1050/7/6/7973 DOI: http://doi.org/10.3390/su7067973

BUSTAMANTE, W.; CEPEDA, R.; MARTÍNEZ, P. \& SANTA MARÍA, H. Eficiencia energética en vivienda social: un desafío posible. En: Camino al Bicentenario. Propuestas para Chile, 2009. pp. 253-282. Santiago, Pontificia Universidad Católica de Chile, Vicerrectoría de Comunicaciones y Asuntos Públicos.

BUSTAMANTE, W.; ROZAS, Y.; CEPEDA, R.; ENCINAS, F.; \& MARTÍNEZ, P. Guía de Diseño para la Eficiencia Energética en la Vivienda Social. Ministerio de Vivienda y Urbanismo; División Técnica de Estudio y Fomento Habitacional y Programa País de Eficiencia Energética, Ed. Santiago, Pontificia Universidad Católica de Chile. 2009.

CALDERA SÁNCHEZ, A. Building Blocks for a Better Functioning Housing Market in Chile. Paris, OECD Publishing. 2012.

CCHC. Mercado Inmobiliario - Oferta Nacional y Gran Santiago. 2017. [En línea] Fecha de consulta: 9 de octubre de 2017. Disponible en: http://www.cchc.cl/centro-deinformacion/indicadores/mercado-inmobiliario-oferta-nacional

CELIS, F.; GARCÍA, R.; TREBILCOCK, M.; ESCORCIA, O.; MIOTTO, U. \& DIAZ, M. Análisis energético de las viviendas del centro-sur de Chile. En: Arquiteturarevista, 2012, 8 (1): 62-75. Disponible en: https://www.redalyc.org/articulo.oa?id=193623828008 DOI: http://doi.org/10.4013/arq.2012.81.07

COLLADOS, E. \& ARMIJO, G. Predicting the impacts of an energy refurbishing programme in Chile: More than energy savings. En: D. Mumovic \& M. Santamouris (Eds.), A handbook of sustainable building design and engineering. An integrated approach to energy, health and operational performance of buildings (First edit). Earthscan Ltd. 2008.

DEFRA. A framework for pro-environmental behaviours. London. 2007.

DITEC. Sistema de calificación energética de viviendas en Chile. Santiago: División Técnica de Estudio y Fomento Habitacional, Ministerio de Vivienda y Urbanismo. 2015.

ENCINAS, F. \& AGUIRRE, C. Sustentabilidad y mercado: aproximaciones desde la promoción inmobiliaria. En: ACE: Architecture, City and Environment, Octubre 2017, 12 (35): 137-164. [En línea] Disponible en: http://revistes.upc.edu/ojs/index.php/ACE/article/view/5141 DOI: http://doi.org/10.5821/ace.12.35.5141

ENCINAS, F.; AGUIRRE, C. \& MARMOLEJO-DUARTE, C. Sustainability Attributes in Real Estate Development: Private Perspectives on Advancing Energy Regulation in a Liberalized Market. En: Sustainability, 2018, 10 (1): 146. [En línea] DOI: http://doi.org/10.3390/su10010146 Disponible en: https://www.mdpi.com/2071-1050/10/1/146

Citación: ENCINAS, F. et al. ¿Cuánto importa el etiquetado energético residencial en los mercados emergentes? Evaluación de las preferencias declaradas sobre sustentabilidad en el mercado inmobiliario de Santiago de Chile En: Libro de proceedings, CTV 2018. XII Congreso Internacional Ciudad y Territorio Virtual. "Ciudades y Territorios Inteligentes". UNCuyo, Mendoza, 5-7 septiembre 2018. Barcelona: CPSV, 2018, p. 285-302. 
ENCINAS, F.; MARMOLEJO, C. \& AGUIRRE, C. El impacto de los proyectos inmobiliarios y sus atributos de sustentabilidad sobre el valor del suelo: ¿causa o consecuencia? Dos estudios de casos para Santiago de Chile. En: Revista Hábitat Sustentable, 6 (2): 70-79. 2016. [En línea] Disponible en: http://revistas.ubiobio.cl/index.php/RHS/article/view/2579

HAIR, J.; ANDERSON, R.; TATHAM, R. \& BLACK, W. Análisis Multivariante (Quinta Edición). Madrid, Pearson Educación, S.A. 2005.

HEINZLE, S. L.; BOEY YING YIP, A. \& LOW YU XING, M. The Influence of Green Building Certification Schemes on Real Estate Investor Behaviour: Evidence from Singapore. En: Urban Studies, 2013, 50 (10): 1970-1987. [En linea] DOI: http://doi.org/10.1177/0042098013477693 Disponible en: https://journals.sagepub.com/doi/abs/10.1177/0042098013477693

ISHIHARA, S. Psychological Methods of Kansei Engineering. En: NAGAMACHI, M. (Ed.), Kansei/Affective Engineering, pp. 31-38. Boca Raton, FL, USA: CRC Press, Taylor and Francis Group. 2011.

JENNINGS, P. \& CAIN, R. A framework for improving urban soundscapes. En: Applied Acoustics, 2013, 74 (2): 293-299. [En línea] DOI: http://doi.org/10.1016/j.apacoust.2011.12.003 Disponible en: https://www.sciencedirect.com/science/article/abs/pii/S0003682X11003082

KANO, M.; SERAKU, N.; TAKAHASHI, F. \& TSUJI, S. ichi. Attractive Quality and Must-Be Quality. En: Journal of the Japanese Society for Quality Control, 1984, 14 (2): 147-156. [En línea] Disponible en: https://link.springer.com/chapter/10.1007\%2F978-3-7908-2380-6_20 DOI: https://doi.org/10.1007/978-3-7908-2380-6_20

KINOSHITA, Y.; COOPER, E. W.; HOSHINO, Y. \& KAMEI, K. A Townscape Evaluation System based on Kansei and Colour Harmony Models. En: 2004 IEEE International Conference on Systems, Man and Cybernetics, pp. 327-332. The Hague, Netherlands. 2004.

LEE, B. J.; JANG, T. Y.; WANG, W. \& NAMGUNG, M. Design Criteria for an Urban Sidewalk Landscape Considering Emotional Perception. En: Journal of Urban Planning and Development, 2009. 135 (4): 133-140. [En línea] Disponible en: https://ascelibrary.org/doi/10.1061/\%28ASCE\%29UP.1943-5444.0000013

LIPPINCOTT, M. Serving the climate-change-conscious consumer. Summary report based on consumer research in the UK and US. 2006.

LIU, F.; Meyer, A. S. \& Hogan, J. F. Mainstreaming Building Energy Efficiency Codes in Developing Countries: Global Experiences and Lessons from Early Adopters. World Bank Working Paper; 204. World Bank. 2010. [En línea] Disponible en: https://openknowledge.worldbank.org/handle/10986/5915

LLINARES, C. \& PAGE, A. Application of product differential semantics to quantify purchaser perceptions in housing assessment. En: Building and Environment, 2007, 42 (7): 2488-2497. Disponible en: https://www.sciencedirect.com/science/article/pii/S0360132306001703 DOI: http://doi.org/10.1016/j.buildenv.2006.06.012

Citación: ENCINAS, F. et al. ¿Cuánto importa el etiquetado energético residencial en los mercados emergentes? Evaluación de las preferencias declaradas sobre sustentabilidad en el mercado inmobiliario de Santiago de Chile En: Libro de proceedings, CTV 2018. XII Congreso Internacional Ciudad y Territorio Virtual. "Ciudades y Territorios Inteligentes". UNCuyo, Mendoza, 5-7 septiembre 2018. Barcelona: CPSV, 2018, p. 285-302. 
LLINARES, C. \& PAGE, A. F. Kano's model in Kansei Engineering to evaluate subjective real estate consumer preferences. En: International Journal of Industrial Ergonomics, 2011, 41 (3): 233-246. [En línea] DOI: http://doi.org/10.1016/j.ergon.2011.01.011 Disponible en: https://www.sciencedirect.com/science/article/abs/pii/S0169814111000187

LÓPEZ-MORALES, E. J.; GASIC KLETT, I. R. \& MEZA CORVALÁN, D. A. Urbanismo proempresarial en Chile: políticas y planificación de la producción residencial en altura en el pericentro del Gran Santiago. En: Revista INVI, 2012. 27 (76): 75-114. [En línea] DOI: http://doi.org/10.4067/S0718-83582012000300003

MARMOLEJO-DUARTE, C., \& VILLAR LLULL, P. El uso de las nuevas técnicas para el análisis de las sensaciones y percepciones que influencian la utilización del espacio público. En: $\underline{A}$. Narváez, G. Vázquez, \& J. M. Fitch (Eds.), Lo imaginario. Seis aproximaciones, pp. 155-191. Monterrey, Mexico: Tilde. 2015.

MARMOLEJO DUARTE, C. \& BRAVI, M. Does the Energy Label (EL) Matter in the Residential Market? A Stated Preference Analysis in Barcelona. En: Buildings, 2017, 7 (2): 53. [En línea] DOI: http://doi.org/10.3390/buildings7020053 Disponible en: https://www.mdpi.com/2075$5309 / 7 / 2 / 53$

MIKULIĆ, J. \& PREBEŽAC, D. A critical review of techniques for classifying quality attributes in the Kano model. En: Managing Service Quality: An International Journal, 2011, 1 (1): 46-66. [En línea] DOI: http://doi.org/10.1108/09604521111100243

MINVU. Informe Técnico Sistema de Calificación Energética de Viviendas. 2017. [En línea] Fecha de consulta: 24 de Octubre de 2017. Disponible en: http://www.calificacionenergetica.cl/media/Informe-Técnico-enero-2017.pdf

MINVU. NTM 011/2 2014 Anteproyecto - Requisitos y mecanismos de acreditación para acondicionamiento ambiental de las edificaciones. Parte 2: Comportamiento higrotémico. 2014 (2014a). Santiago, Ministerio de Vivienda y Urbanismo

MINVU. Política Nacional de Desarrollo Urbano. Ciudades Sustentables y Calidad de Vida. 2014 (2014b). J. R. Giménez, Pilar; Ugarte, Ed. Santiago: Ministerio de Vivienda y Urbanismo

MINVU. Ordenanza General de Urbanismo y Construcciones. 2016. Santiago: Ministerio de Vivienda y Urbanismo.

NAGAMACHI, M. Kansei Engineering: A new ergonomic consumer-oriented technology for product development. En: International Journal of Industrial Ergonomics, 1995, 15 (1): 3-11. Disponible en: https://www.sciencedirect.com/science/article/abs/pii/0169814194000525 DOI: http://doi.org/10.1016/0169-8141(94)00052-5

NAGAMACHI, M. Methods of Kansei/Affective Engineering and Specific Cases of Kansei Products. En: M. Nagamachi (Ed.) Kansei/Affective Engineering, 2011. pp. 13-29. Boca Raton, FL, USA: CRC Press, Taylor and Francis Group. 
OFFICIAL JOURNAL OF THE EUROPEAN UNION. Directive 2002/91/EC of the European Parliament and of the Council of 16 December 2002 on the energy performance of buildings. 2003.

POORTINGA, W.; STEG, L.; VLEK, C. \& WIERSMA, G. Household preferences for energysaving measures: A conjoint analysis. En: Journal of Economic Psychology, 2003, 24 (1): 4964. Diponible en: https://www.sciencedirect.com/science/article/abs/pii/S016748700200154X DOI: http://doi.org/10.1016/S0167-4870(02)00154-X

SADLER, M. Home energy preferences \& policy: Applying stated choice modeling to a hybrid energy economy model. School of Resource and Environmental Management, Simon Fraser University. 1998.

SAVAGE, M.; Warde, A. \& Ward, K. Urban Sociology, Capitalism and Modernity (Second edi). Palgrave Macmillan. 2003.

TAMA, I. P.; AZLIA, W. \& HARDININGTYAS, D. Development of Customer Oriented Product Design using Kansei Engineering and Kano Model: Case Study of Ceramic Souvenir. En: Procedia Manufacturing, 2015, 4(less):328-335. DOI: http://doi.org/10.1016/j.promfg.2015.11.048 Disponible en: https://www.sciencedirect.com/science/article/pii/S2351978915011646

VIOLANTE, M. G. \& VEZZETTI, E. Kano qualitative vs quantitative approaches: An assessment framework for products attributes analysis. En: Computers in Industry, 2017, 86, 15-25. Disponible en: https://www.sciencedirect.com/science/article/pii/S0166361516303566 DOI: http://doi.org/10.1016/j.compind.2016.12.007

Citación: ENCINAS, F. et al. ¿Cuánto importa el etiquetado energético residencial en los mercados emergentes? Evaluación de las preferencias declaradas sobre sustentabilidad en el mercado inmobiliario de Santiago de Chile En: Libro de proceedings, CTV 2018. XII Congreso Internacional Ciudad y Territorio Virtual. "Ciudades y Territorios Inteligentes". UNCuyo, Mendoza, 5-7 septiembre 2018. Barcelona: CPSV, 2018, p. 285-302. 